2. A uf Pharmazie bezügliche Methoden. Yon

\title{
H. Mühe.
}

Über die Bestimmung des Chinins in Gemischen der Chinaalkaloide, in der Chinarinde und in galenischen Präparaten berichtet W. Hill $\mathrm{e}^{1}$ ) in einer ausführlichen Abhandlung. Der Verfasser hat die Methoden der Chininbestimmung einer kritischen Prüfung unterzogen und bei der Nachprüfung der ältesten Methode, der Trennung durch Behandeln mit Äther, gefunden, dass Chinin durchaus nicht so leicht löslich in reinem Äther ist, wie gewöhnlich angenommen wird; es ist sogar schwerlöslich. Seine Löslichkeit ist je nach der angewandten Ätherart verschieden. Sie betrug bei Verwendung von absolutem Äther $1: 46,5$, bei Verwendung von solchem, welcher $4 \%$ Alkohol enthielt, $1: 10$. Ein grösserer Zusatz von Weingeist bewirkte keine grössere Löslichkeit des Chinins. Beim Ausschütteln von Chinin mittels eines solchen alkoholhaltigen Äthers aus alkalischer Lösung erhält man eine Flüssigkeit, die gar nicht den Eindruck einer Lösung macht. Zum Vertreiben des Äthers muss man eine Temperatur von $90-100^{\circ}$ anwenden, wobei eine gelatinöse Mischung hinterbleibt, die erst bei 100 bis $110^{\circ}$ reines Chininanhydrid liefert. Die Löslichkeit des Chinins in Äther, der mit Wasser gesättigt ist, fand der Verfasser zu $1: 19,8$.

Weiterhin zog Hille die folgenden Methoden in den Bereich seiner Prüfung: die Herapathitmethode von de Vrij; die Oxalatmethode von Shimoyama; die Polarisationsmethode; die Sulfatmethode von P. Carles; die Tartratmethode von H. Schmidt; die Einwirkung von Jodkalium auf die Lösungen von Chinaalkaloidsalzen; die Löslichkeit der Nitroprussidsalze der Alkaloide; die Chromatmethode; die Trennungsmethode mit Hilfe der benzolthiosulfosauren Salze der Chinaalkaloide und die quantitative Bestimmung des Chinins mit Hilfe von Äther, welcher mit den Nebenalkaloiden gesättigt ist.

Hille empfiehlt nun folgende Methoden zur Bestimmung des Gesamtalkaloidgehaltes und des Chiningehaltes der Chinarinde.

1. Sulfatmethode. Man übergiesst $12 \mathrm{~g}$ feines, bei $100^{\circ}$ getrocknetes Chinarindenpulver in einem Arzneiglase mit $90 \mathrm{~g}$ Äther und $30 \mathrm{~g}$ Chloroform, versetzt die Mischung mit $10 \mathrm{cc}$ Natronlauge und lässt unter häufigem, kräftigem Umschütteln 3 Stunden lang stehen. Hierauf fügt

1) Archiv d. Pharmazie 241, 54; durch Pharm. Zentralhalle 45, 79. 
man so viel Wasser (etwa $10 c c$ ) hinzu, dass sich das Chinarindenpulver beim kräftigen Schütteln zusammenballt und die darüber stehende Chloroform-Ätherschicht sich völlig klärt. Nach einstündigem Stehen filtriert man $100 \mathrm{~g}$ (= $10 \mathrm{~g}$ Rinde) davon durch ein trockenes Filter in ein Kölbchen, verdunstet das Chloroform und den Äther und trocknet bei etwa $110^{\circ}$; der Rückstand soll wenigstens $0,5 \mathrm{~g}$ betragen.

Den im Kölbchen enthaltenen Rückstand bringt man nun mit Wasser und verdünnter Schwefelsäure in Lösung, filtriert und spült das Gefäss noch dreimal mit schwefelsäurehaltigem Wasser nach. Das klare Filtrat bringt man mit Wasser auf etwa $50 \mathrm{cc}$ und neutralisiert (genau) siedendheiss mit Ammoniakflüssigkeit. Nach sechsstündigem Stehen sammelt man die flockigen Ausscheidungen auf einem gewogenen Filter, wäscht das Filter mit $20 c c$ Wasser aus und trocknet bei höchstens $110^{\circ}$; dem gefundenen Gewichte addiert man $0,0078 \mathrm{~g} \mathrm{zu}$ und rechnet es mit $\frac{648}{746}$ auf Chinin um; das Gewicht soll dann mindestens $0,2 g$ betragen.

2. Anwendung des Ätherverfahrens zur quantitativen Bestimmung des Chinins in der Chinarinde. Nach dem Wägen der wie oben erhaltenen Gesamtalkaloide ubergiesst man das Alkaloidgemisch mit $50 \mathrm{cc}$ einer Flüssigkeit, bestehend aus Chinidin 2,44 g, Cinchonidin 1,45 g, Cinchonin $0,14 g$, Äther $96 g$ und Spiritus $4 g$, verschliesst das Kölbchen, schüttelt es während einer Stunde häufig um und stellt es einen Tag lang in den Keller beiseite. Nachdem man die Temperatur der Flüssigkeit gemessen hat, verdunstet man $25 c c$ der klaren Lösung in einem gewogenen Becherglase und trocknet bei $125-135^{\circ}$. Von dem Gewicht der Alkaloide zieht man die für die betreffende Temperatur angegebene Zahl für $25 c c$ ab und verdoppelt das so erhaltene Resultat, um das Gewicht des Chinins zu ermitteln. Auf die vom Verfasser beigefügte Tabelle, aus welcher die Korrekturen für die Intervalle von $+20^{\circ}$ bis $+8^{0}$ ersichtlich sind, kann ich hier nur hinweisen.

Für die Bestimmung des Gesamtalkaloidgehaltes und des Chinins in Extraktum chinae aquosum gibt Hille folgendes Verfahren an: man löst $3 g$ wässriges Extrakt in einem Messzylinder mit Hilfe von $5 g$ Wasser und $5 g$ absolutem Weingeist, fügt $50 \mathrm{cc}$ Äther und $10 c c$ Chloroform, sowie nach kräftigem Umschütteln $10 c c$ Natriumkarbonatlösung (1:3) hinzu und lässt die Mischung hierauf unter häufigem Umschütteln drei Stunden lang stehen. Nachdem sich die Schichten getrennt haben, fällt man die Ätherschicht mit Äther $\mathrm{zu} 75 \mathrm{cc}$ auf, verdunstet $50 \mathrm{cc}$ 
derselben und trocknet den Rückstand bei $105^{\circ}$; er soll wenigstens $0,12 g=6 \%$ Gesamtalkaloidgehalt betragen. Die Chininbestimmung führt man nach einer der beiden oben besprochenen Methoden aus: Auf die gleiche Weise kann man Extractum chinae spirituosum, Tinctura chinae und Tinctura chinae composita auf ihren Gesamtalkaloid- und Chiningehalt prüfen; bei den Tinkturen verwendet man $50 \mathrm{~g}$ und dampft auf $10 \mathrm{~g}$ ein.

Über die Unterscheidung des Chloralhydrats vom Butylchloralhydrat veröffentlicht E. Gabutti ${ }^{1}$ ) die Resultate seiner Versuche. Als Reagens empfiehlt der Verfasser eine Lösung von Pyrogallussäure in reiner konzentrierter Schwefelsäure. Versetzt man die Chloralhydrate mit diesem Reagens, so erhält man ungefärbte Reaktionsflüssigkeiten, während bei gelindem Erwärmen das Chloralhydrat eine schön blaue, das Butylchloralhydrat aber eine schön weinrote Farbe annimmt. Verdünnt man nun mit viel Wasser, so geht das Blau in Gelbbraun über, das Weinrot des Butylchlorals gibt je nach der zugesetzten Wassermenge ein mehr oder weniger blasses Violett. Liegen beide Chloralhydrate gemischt vor, so treten die Farbenerscheinungen dem entsprechend verwischt auf, man muss alsdann Kontrollversuche mit Gemischen von bekannter Zusammensetzung ausführen.

Über die Löslichkeit des Natriumbromides in Weingeist berichtet J. D. Riedel ${ }^{2}$ ). Das D. A. B. IV sagt vom Natrium bromatum: Weisses kristallinisches Pulver, in 5 Teilen Weingeist löslich; 100 Teile enthalten mindestens 95 Teile wasserfreies Salz. Riedel weist darauf hin, dass ein völlig wasserfreies Natriumbromid 9,2 Teile und solches mit $5 \%$ Wassergehalt, wie das D. A. B. IV es gestattet, 9 Teile offizinellen Weingeist zur Lösung verlangt; wenn nun das D. A. B. IV von 5 Teilen als genügender Lösungsmenge spricht, so ist dieses Lösungsverhältnis unrichtig; wie der Verfasser nachgewiesen hat, löst sich nur das kristallwasserhaltige Natriumbromid, welches $74,1 \%$ wasserfreies Salz enthält, in 5 Teilen Weingeist von 90 Volumprozent.

Über die Prüfung von Zinkoxyd auf Magnesia macht Jorissen ${ }^{3}$ ) Mitteilung. Das D. A. B. IV sagt von der Prüfung des Zinkoxydes: Zinkoxyd soll sich in 10 Teilen verdünnter Essigsäure ohne Aufbrausen lösen. Diese Lösung soll, mit überschüssiger Ammoniakflüssigkeit ver-

1) Boll. Chim. Pharm. 1903, No. 24; durch Pharm. Zentralhalle 45, 129.

2) Pharm. Zentralhalle 45, 95.

3) Journal de Pharm. d'Anvers 1903, S.381; durch Pharm. Zentralhalle 45, 97. 